\title{
Decision Support Procedure for Medical Equipment Maintenance Management
}

\author{
Malek Masmoudi, Zeineb Ben Houria, PhD, Ahmad Al Hanbali, and Faouzi Masmoudi
}

\begin{abstract}
Hospitals outsource several activities of the service support in order to focus on the core healthcare production as maintenance service. Recently, faced to the sophistication and the costs of medical equipment that continue to escalate, governments have implemented new reforms to control costs and improve the efficiency and the quality. Hospitals become interested in minimizing the total operational cost, by optimizing healthcare production planning and their support activities. Reorganizing the medical equipment maintenance service becomes a priority for the hospital managers to reduce the cost and the dependency on external parties while ensuring that the medical devices are safe, accurate, and operating at the required level of performance. In this article, we propose an efficient procedure to take the appropriate decisions for medical equipment maintenance such as the selection of maintenance strategy, the insourcing/outsourcing, and the selection of contracts' type and content. A practical application of this procedure in the Tunisian context is considered. Nevertheless, our procedure is general and can be tailored to hospitals in both developed and developing countries.
\end{abstract}

Corresponding author: Malek Masmoudi, is assistant professor in industrial engineering at the University of Jean-Monnet, Saint Etienne in France. He obtained his doctor of philosophy degree in industrial engineering from the University of Toulouse in France. He can be reached at malek.masmoudi@univ-st-etienne.fr.

Zeineb Ben Houria, PhD, is a student of doctor of philosophy degree at National school of Engineers of Sfax at the University of Sfax in Tunisia. She is working on the optimization of the maintenance of medical equipments with a collaborative project with Habib Bouguiba Tunisian Hospital. Her research interests include maintenance optimization models and mathematical modeling. She received her bachelor of science degree in industria engineering from the National School of Engineers of Bizerta, Tunisia.

Ahmad Al Hanbali, is associate professor in industrial engineering and business information systems at the University of Twente, Enschede, the Netherlands. He is a full member of the Beta Research School, the Netherlands; the International Society of Inventory Research; Twente Is Maintenance Excellence; and Service Logistics Forum. He was a member of many technical program committees of international conferences. He served as a reviewer for several international journals.

Faouzi Masmoudi, is full professor at the National School of Engineers of Sfax in Tunisia and a researcher at the Mechanics Modelling and Production Research Unit (U2MP). He obtained his doctor of philosophy degree in computer-integrated manufacturing (1988) from ENSAM, Paris, France.

The authors declare no conflicts of interest.

DOI: 10.1097/JCE.0000000000000135
Modern medical devices and equipment have become very complex and sophisticated and are expected to operate under stringent environments. Hospitals must ensure that their critical medical devices are safe, accurate, reliable and operating at the required level of performance. ${ }^{1}$ According to Khalaf, ${ }^{2}$ the greatest problem for many developing countries is not the lack of equipment, but rather that $50 \%$ and sometimes up to $75 \%$ of the equipment supplied is not operative. As cited by the Centre for Biomedical Engineering and Hospital Maintenance, ${ }^{3}$ the main purpose of a biomedical maintenance service is to ensure patient and user safety by maintaining an optimal performance for all biomedical devices.

The complexity and the cost of biomedical maintenance have risen sharply in the last few decades. In fact, a maintenance activity is of different types:

- Corrective maintenance: This activity is performed after detecting a failure and intended to return the medical device to an operational state. ${ }^{4}$ According to Rahman and Chattopadhyay, ${ }^{5}$ the strategy of running the equipment until failure does not require any extra labor or a special budget to be applied. Yet, the random downtime and the overuse of the labor to repair the equipment can be costly.

- Preventive maintenance: This activity is performed at predetermined intervals or according to prescribed criteria and intended to reduce the probability of failure or the degradation of medical equipment. ${ }^{6}$ Preventive maintenance is considered to be potentially effective according to Gits ${ }^{7}$ when the equipment suffers from wear-out phenomenon; that is, it has an increasing failure rate. We distinguish 2 categories of preventive maintenance activities:

Time-based maintenance (TBM): In this category, the equipment is periodically checked and maintained. This activity can reduce the failure rate of equipment. However, it requires specific resources such as budget and labor to be implemented. ${ }^{6}$ Condition-based maintenance (CBM): It requires regular assessment of the system condition during operation. For this category, we need special sensors to measure the prediction variables. ${ }^{5}$ Moreover, a statistical model is required to relate the measured variables to the equipment (state) health, for example, equipment remaining useful life. 
- Quality control: assessment of the equipment performance through inspections and how they are planned precisely ${ }^{8}$

- Selection and monitoring of different contracts with suppliers, subcontractors, and service companies

- Making recommendations for the purchasing of new devices and the training of hospital personnel

Maintenance is characterized by the plurality of tasks that can be different in nature and durations. These tasks are grouped into 5 levels, ${ }^{8}$ depending on where they can be carried out (in-house vs outside the hospital) and by which party (internal vs external resources). In addition for these tasks, the complexity and the tools required play a determinant role in the grouping. Levels 1 and 2 are carried out in-house; level 1 by internal resources and level 2 by internal and/or external resources. Level 3 and 4 are carried out inside or outside by internal or external resources. Level 5 is for rare complex tasks and is carried out by the Original Equipment Manufacturers (OEM). In the biomedical maintenance service, there are different human resources, referred to as staff that include operators and engineers. They are considered competent per equipment and per maintenance level if applicable.

For many healthcare institutions in developing countries, contracting maintenance services is inevitable because they often have neither properly trained staff nor the required material to handle the maintenance tasks on their own. ${ }^{9}$ In fact, the in-house maintenance needs special tools and test equipment that may not be available or may need additional training costs, especially for the staffs who are typically generalist rather than specialist. ${ }^{10}$

Three service/support options are possible for maintaining the medical equipment: in-house biomedical engineering service, OEM, and third-party service provider (with or without contracts). Each contract contains several clauses that formalize the relationship between the in-house biomedical service and the service provider (third-party provider or OEM). The estimation of costs for such contracts is a challenging task, but it is really important to the biomedical maintenance service and the service providers (OEM or third party) for economic reason. ${ }^{5}$

According to Cruz et al, ${ }^{11}$ the most desirable clauses featured in any contract between a hospital and a service provider include repair responsibility, parts and tools, response time, equipment uptime, maintenance and repair log, penalties in case of underperformance, price of tasks, and payment terms.

The possible types of contracts differ from one country to another. ${ }^{18}$ In developing countries, medical equipment maintenance is costly and partially mastered most of the time because it is usually managed by external service contracts. ${ }^{9}$ According to Letaief et $\mathrm{al}^{3}{ }^{3}$ the possible contracts in the Tunisian context are as follows:
- Contract type A: All tasks of TBM strategy are performed by the subcontractor with labor and spare parts included in the maintenance package.

- Contract type B: In this type, the TBM and/or CBM tasks are performed by the subcontractor with only spare parts included in the package.

- Contract type C: For this contract, there is no package offered. In general, it is used for corrective maintenance when failures are complex.

In practice, a fourth type of contract is often preferred by the in-house biomedical engineering service:

- Contract type $\mathrm{A}^{*}$ : It covers all risks. All maintenance tasks (corrective and preventive) are performed by the subcontractor and included in the maintenance package.

Some clauses in these contracts are the same as, for example, the intervention duration, the response time, and the equipment downtime. The monitoring is done with a dashboard that contains these performance indicators.

The remainder of this article is structured as follows: In section 2, we present the state of the art. In section 3, a 4-step decision support procedure is proposed and explained in details. In section 4 , the conclusions and the future perspectives of this study are made.

\section{State of the Art}

According to the World Health Organization, ${ }^{12}$ the major challenge of biomedical maintenance service is to implement a maintenance strategy, which maximizes availability and efficiency of the equipment, controls the rate of equipment deterioration, ensures safety, and minimizes the total costs.

The majority of studies focus on the reliability ${ }^{13}$; the criticality of medical equipment as reported by Fennigkoh and Smith, ${ }^{14}$ Wang and Levenson, ${ }^{15}$ and Mkalaf et $\mathrm{al}^{4}$; and the planning of maintenance strategies. Jamshidi et $\mathrm{al}^{16}$ provided a literature review on medical devices inspection and maintenance and divided the studies (academic journal articles published between 1985 and 2014) into 3 main categories: the prioritization of medical devices (to restrict the maintenance planning on high-priority devices), the empirical research comparing different case studies, and the mathematical modeling and optimization of maintenance planning. According to Khalaf et al, ${ }^{17}$ very little analysis has been performed to measure and monitor the effectiveness of the maintenance strategies including a revision to maximize effectiveness, increase reliability, and optimize costs.

The idea of prioritization of medical equipment is to allow managers with a limited budget and resources to give priority to a limited portion of medical equipment that are critical. Fennigkoh and Smith ${ }^{14}$ proposed a "risk-based 
criteria" and identified 3 main subfactors: equipments' function (therapeutic, diagnostic, analytical, and miscellaneous), physical risk to patients, and maintenance requirements. According to Fennigkoh and Smith, ${ }^{14}$ there are 3 classes of maintenance requirements: extensive maintenance for equipment that is predominantly mechanical, pneumatic, or fluidic; average maintenance requirement if equipment requires only performance verification and safety testing; and minimal maintenance requirements for equipment that receives only visual inspection or basic performance check. The calculation of the overall equipment management (EM) risk factor is defined as follows in Equation 1:

$$
\mathrm{EM}=\text { function }+ \text { risk }+ \text { maintenance requirements }
$$

Levels are associated to each subfactor according to its importance. Then, devices with an EM above a threshold, for example, larger than 12, are considered to be critical and thus included in the inspection and the maintenance planning. The detailed definition of these subfactors is given in First Step: Calculating the Equipment's Criticality.

Wang and Levenson ${ }^{15}$ proposed a new interpretation of the function subfactor and called it mission criticality. They defined this subfactor as the equipment role or importance within the global healthcare organization's mission as opposed to the individual clinical department. Then, they introduced the utilization rate of equipment, that is, the average percentage of time each type of equipment is being used. They argued that a low utilization rate yields not only less urgency for equipment repairs but also less criticality for the equipment. The new formulation of the adjusted equipment management rating (EMR) is a weighted sum of the following subfactors (Equation 2):

Adjusted $\mathrm{EMR}=($ mission critical $+2 *$ maintenance requirements $)$

$$
\text { *utilization rate }+2 * \text { risk }
$$

A multicriteria decision-making model to prioritize medical equipment according to their criticality is presented by Taghipour et al. ${ }^{6}$ They used the analytical hierarchy process and proposed a sequence of weighted criteria to define the criticality of the equipment. Moreover, they described how individual scores obtained for each criterion can be used to establish a guideline for the selection of appropriate maintenance strategies. Jamshidi et $\mathrm{al}^{16}$ proposed a fuzzy healthcare failure modes and effects analysis method for the prioritization of medical devices. They calculated and prioritized the risk based on the conditional probability of failures and their consequence.

Several articles discuss the insourcing and outsourcing decision together with the choice of the appropriate maintenance contract. ${ }^{18} \mathrm{~A}$ mapping review of 213 papers is provided by Cruz and Rincon, ${ }^{9}$ containing mathematical models for decision making in maintenance outsourcing, contract design, negotiation, and optimization. The outsourcing of maintenance tasks has advantages and disadvantages that can be identified according to human resources, material resources, and the overall cost of the activity. ${ }^{18}$ The decision to outsource depends on the availability of appropriate skills and tools in-house. Other criteria are considered and related to equipment, hospital, and subcontractor. We conclude that the research into the outsourcing of medical device maintenance services and its associated risks in hospitals is still in its infancy stages. ${ }^{16}$

A contract is usually signed for more than 1 year and managed by the in-house service in coordination with the subcontractor. The contract monitoring (assessment of the subcontractor) is crucial to determine the maintenance costs (including price and penalties). Georgin et $\mathrm{al}^{18}$ provided a procedure to select a contract type per equipment. The considered selection criteria are mainly the availability of resources, the possibility of training, the availability of tools, the cost of spare parts, and the mean time between failures. A new type of contract called "partnership contract" is proposed in the procedure. This type of contract is the subject of several studies in the literature and already applied in several hospitals in developed countries. ${ }^{10,18}$ This type of contract is not available in the Tunisian context.

We conclude that the literature on medical equipment maintenance planning is scattered into different categories. Our objective in this article is to provide a unifying approach that integrates the most important decisions of medical equipment maintenance.

\section{Decision Support Procedure}

To the best of our knowledge, this is the first article that proposes a decision support procedure to select the appropriate maintenance strategy, to choose to insource/ outsource and to select the appropriate contract.

A proposal model for a hierarchical decision support procedure is shown in the Figure. This procedure is inspired by a case study done at a Tunisian hospital, discussions with hospital experts, and an extensive study of the state of the art.

According to Panayiotou et $\mathrm{al},{ }^{19}$ the development of a suitable maintenance procedure allows to take the decision of maintenance strategies based on the existing factors. For that reason, our provided decision support procedure is based on the following technical, financial, human, and organizational criteria:

- availability of maintenance tools

- availability of competent staff

- equipment criticality factor

- maintenance load time and cost

- complexity and frequency of failures

- costs of spare parts 


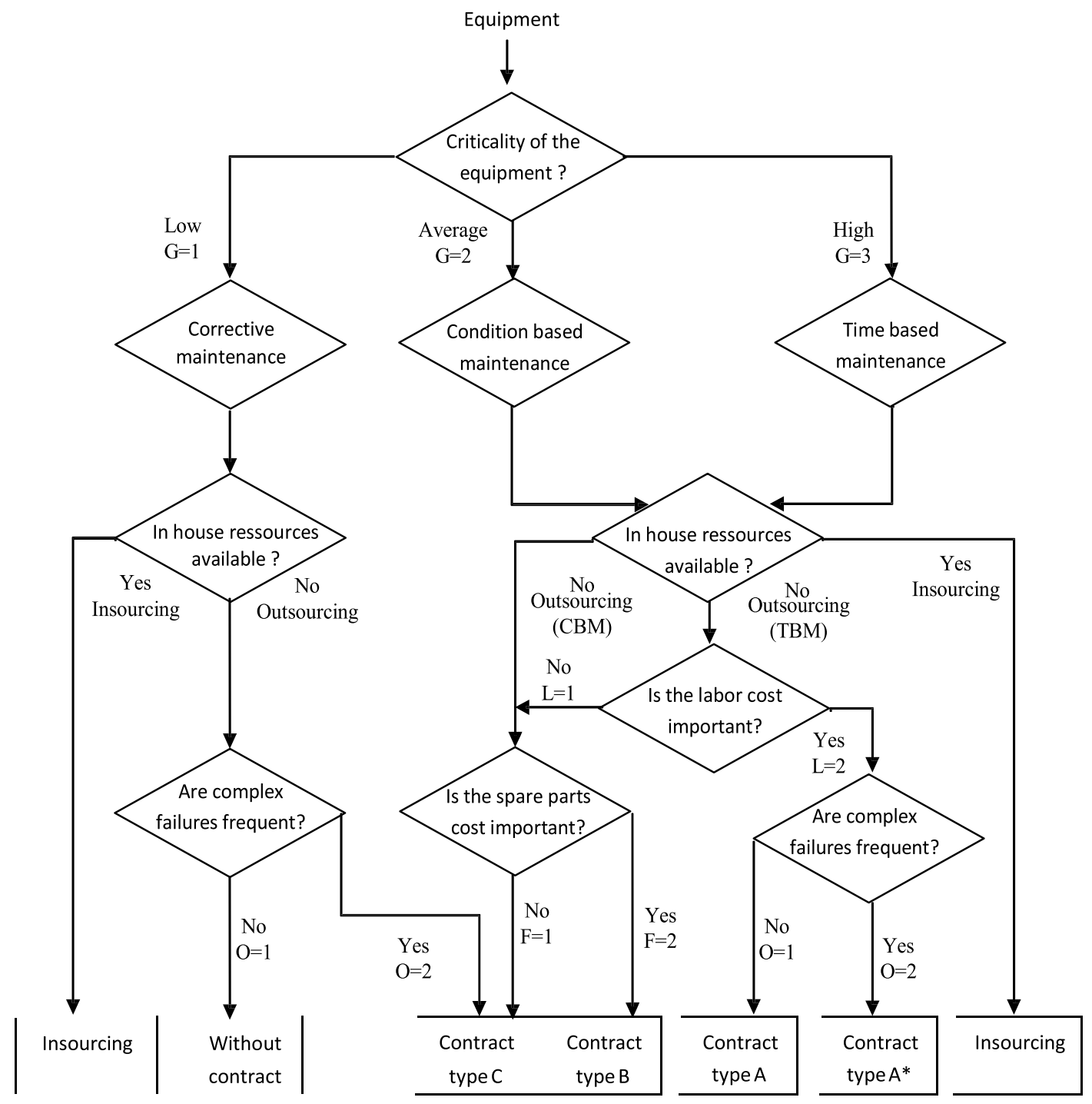

FIGURE. Diagram of decision support of equipment maintenance management details on parameters (G values explained in subsection; First Step: Calculating the Equipment's Criticality L values explained in subsection. Second Step: Insourcing Based on a Heuristic to Set Up the Maintenance Service Workload and F, and O values explained in section. Fourth Step: Outsourcing and Setting Up the Contract).

The main idea behind our procedure is that preventive maintenance strategy will be mainly applied for the critical equipment. Moreover, it is cheaper and more beneficial to insource a maintenance activity as long as the required resources are available internally. Last, the type of a maintenance contract should depend on the complexity of repair tasks and the price of spare parts. Motivated by these facts, we propose the following sequential 4-step procedure:

- Step 1: Calculating the equipment's criticality using a multicriteria analysis

- Step 2: Insourcing decision by applying a simple heuristic that checks the feasibility based on the internal constraints and costs

- Step 3: Selecting the maintenance strategy per equipment based on the resources availability and the budget
- Step 4: Outsourcing the rest with or without a contract and choosing the convenient type of contract based on spare parts cost and repairs complexity

These 3 steps are detailed in Section 3.1, 3.2, 3.3 and 3.4 respectively.

First Step: Calculating the Equipment's Criticality Taghipour et $\mathrm{al}^{6}$ proposed a multicriteria decision-making model to determine the criticality of medical devices using the aforementioned 5 criteria. Moreover, they have added in particular the maintenance costs (labor, tools, and spare parts) as an additional criterion. Based on a descending order of criticality, they have considered the following order of maintenance policies: condition-based preventive maintenance for highly critical equipment, time-based preventive 


\begin{tabular}{|c|c|c|}
\hline Criteria & No. of Subcriteria & No. of Levels \\
\hline $\begin{array}{l}\text { Degree of complexity of } \\
\text { the maintenance }(A)\end{array}$ & - & 3 \\
\hline Function (B) & - & 9 \\
\hline Risk (C) & 4 & 3 \\
\hline $\begin{array}{l}\text { Degree of the mission } \\
\text { importance }(D)\end{array}$ & 2 & 3 \\
\hline Age (E) & - & 2 \\
\hline
\end{tabular}

maintenance for medium critical equipment, and corrective maintenance for noncritical equipment. There is an issue regarding the applicability of this ordering in our procedure: the cost criterion is perfectly in the direction of the considered classification "condition-based, time-based, and corrective." However, the function criterion that has the most important weight is in the direction of the subsequent classification "time based, condition based, and corrective."

Several methods are developed in the literature for determining equipment's criticality. In our model, we calculate the criticality based on 5 criteria (degree of maintenance complexity, function, risk, level of importance of the mission, and age) and 6 subcriteria (detectability, frequency of failures, safety, downtime, utilization rate, and availability of alternative devices).

According to the hospital experts, the classification "time-based preventive, condition-based preventive, and corrective" is more appropriate. Therefore, we considered this classification and excluded the cost criteria (labor, tools, and spare parts) from the calculation of equipment criticality. The cost criterion is kept for the following steps in the decision procedure. In addition, instead of using the weights for the criteria, we decided to adjust the criterion levels in the following way. For a criterion with a level from 1 to 5 and a weight of 2 as used by Taghipour et al, ${ }^{6}$ we consider 10 levels from 2 to 10 without weights. This way is practical (easy to implement) in the hospital where thousands of equipment are included in the study. Thus, we adopted for these ideas and confirmed their validity with hospital's experts. Table 1 shows the criteria and their levels. In the following, we will explain in details the different criteria and the subcriteria together with their associated levels.

\section{Degree of Complexity of the Maintenance (A)}

Fennigkoh and Smith ${ }^{14}$ considered the degree of complexity of the required maintenance as a criterion to calculate the criticality of a medical device. Five levels are associated to this criterion according to its importance. For easiness, we propose 3 levels with a score from 1 to 3 :

- high complexity of the required maintenance (score $=3$ ): advanced mechanical equipment, pneumatic or hydraulic

- medium complexity of the required maintenance $($ score $=2)$ : equipment that requires only verification of performance and safety tests

- low complexity of the required maintenance (score $=1$ ): equipment that receives only visual inspections

\section{Function (B)}

We define the function of a medical device as the primary purpose for which it is used. Fennigkoh and Smith ${ }^{14}$ proposed a score from 1 to 9 to this criterion. In our model, we use the same definition and score (Table 2).

\section{Risk (C)}

Risk is the most important criterion for determining the criticality of a medical device but cannot be simply considered as a single number assigned to a device. ${ }^{6}$ Several methods are used for its determination, in particular, failure mode and effects analysis. Taghipour et $\mathrm{al}^{6}$ have used the detectability, the frequency of occurrence of failures, and their consequences in terms of cost, safety, and downtime. In our study, we consider these subcriteria except consequences in term of costs and consider 3 levels per each subcriterion.

The risk is the sum of the individual failure modes risks (FMRs). ${ }^{6}$ In our study, to calculate each FMR, we consider the following Equation 3:

$\mathrm{FMR}=$ detectability $*$ frequency $*($ downtime + safety $) / 2$

As each subcriterion (detectability, frequency, downtime, and safety) allows 3 levels (high, medium, and low),

\begin{tabular}{|l|l|c|}
\hline TABLE 2. Equipment Function & \\
\hline Classes & & Score \\
\hline \multirow{3}{*}{ Therapeutic } & Life support & 9 \\
\cline { 2 - 3 } & Surgical and intensive care & 8 \\
\cline { 2 - 3 } & Physical therapy and treatment & 7 \\
\hline \multirow{2}{*}{ Diagnostic } & Surgical and intensive care monitoring & 6 \\
\cline { 2 - 3 } & $\begin{array}{l}\text { Additional physiological monitoring } \\
\text { and diagnostic }\end{array}$ & 5 \\
\cline { 2 - 3 } & Analytical laboratory & 4 \\
\cline { 2 - 3 } & Laboratory accessories & 2 \\
\cline { 2 - 3 } & Computers and related & 1 \\
\hline \multirow{2}{*}{ Miscellaneous } & Patient related and other & \\
\hline
\end{tabular}


FMR is an integer value between 1 and 27 . Then, the risk is an integer value from 1 to 27 multiplied by the maximum number of failure modes among all equipment under study. For example, for a medical device with 10 failure modes, risk is between 10 and 270 .

Nevertheless, instead of calculating risk values, we propose a heuristic reasoning to deduct the 3 levels for risk: high $($ score $=3)$, medium $($ score $=2)$, and low $($ score $=1)$, with respect to its subcriteria's levels assigned to that device. A deterministic reasoning such as propositional logic or a nondeterministic reasoning like fuzzy logic can be used. ${ }^{20}$ In this case, the rules of inference should be established before assessing the alternatives. Rules are knowledge expressions in the form of conditions and actions. A rule consists of an if statement and a then statement, for example, "If detectability is high, and frequency is medium, and downtime is medium, and safety is high, then risk is high."

\section{Degree of the Mission Importance (D)}

Wang and Levenson ${ }^{15}$ considered the degree of mission importance or the mission criticality as a criterion to calculate the device's criticality. This criterion describes the extent to which a device is crucial to the care delivery process of a hospital. In our model, the degree of importance of the mission depends on the utilization rate (the utilization rate is considered as the average hours of equipment usage per week divided by the maximum, which is considered 48 hours per week) and the availability of alternative devices. ${ }^{6}$ We consider 4 scores for the utilization rate and 2 scores for the availability of alternative devices (Table 3).

We consider a simple sum of the 2 subcriteria (S) to calculate the degree of importance of the mission criterion. The value of $S$ is between 2 and 6. In our model, we propose 3 scores for this criterion: high importance (score $=3$ ), medium importance $($ score $=2$ ), and low importance $($ score $=1)($ Table 4$)$.

\begin{tabular}{|l|l|c|c|}
\hline TABLE 3. & \multicolumn{3}{|c|}{$\begin{array}{l}\text { Subcriteria to Calculate Degree of } \\
\text { Importance }\end{array}$} \\
\hline Criteria & \multicolumn{1}{|c|}{ Subcriteria } & Description & Score \\
\hline $\begin{array}{l}\text { Degree of } \\
\text { importance }\end{array}$ & $\begin{array}{l}\text { Utilization } \\
\text { rate (UR) }\end{array}$ & UR $\geq 80 \%$ & 4 \\
\hline \multirow{2}{*}{} & & $65 \% \leq \mathrm{UR}<80 \%$ & 3 \\
\cline { 3 - 4 } & & $30 \% \leq \mathrm{UR}<65 \%$ & 2 \\
\cline { 3 - 4 } & $\begin{array}{l}\text { Availability of } \\
\text { alternative devices }\end{array}$ & Not available $<30 \%$ & 2 \\
\cline { 3 - 4 } & & Available & 1 \\
\hline
\end{tabular}

\begin{tabular}{|l|c|c|}
\hline TABLE 4. Degree of Importance of the Mission \\
\hline Classes of the Degree of Importance & $\mathrm{S}$ & Score \\
\hline High & $\mathrm{S}=6$ & 3 \\
\hline Medium & $\mathrm{S} \in\{4,5\}$ & 2 \\
\hline Low & $\mathrm{S}\{2,3\}$ & 1 \\
\hline
\end{tabular}

\section{Age (E)}

Age score is based on the actual age of a device and its predictable lifetime that is usually considered equal to 10 years. $^{6}$ In our model, we propose 2 levels: new (when age $\leq 10$ years, score $=1$ ) and old (when age $>10$ years, score $=2$ ).

\section{Criticality (G)}

We propose a simple formula with a summation of the last 5 criteria (from A to E) to calculate the equipment criticality value. We do not consider weight because the importance of each criterion is already considered by the number of associated levels.

In our model, the total score of criticality is between 5 and 20. Table 5 shows an example of high critical equipment.

\section{Second Step: Insourcing Based on a Heuristic to Set Up the Maintenance Service Workload}

The decision on whether to conduct the medical device maintenance in-house or to outsource is considered based on additional criteria: competence of the internal staff and availability of necessary tools, (average) estimated annual cost, required maintenance team, and cost of labor externally. Because the equipment maintenance strategy is not yet known in this step, we take the average cost of the 3 possible strategies as estimation.

\section{Competence per Level of Maintenance $(H)$}

According to the Norme NF-X60-010, ${ }^{21} 5$ levels of maintenance from 1 to 5 are defined. Staff competence will be identified by level. Level L1 corresponds to simple

\begin{tabular}{|l|l|c|}
\hline \multicolumn{2}{|c|}{ TABLE 5. Criticality Study of the Anesthesia } \\
Ventilator Type Dräger Julian \\
\hline Criteria & \multicolumn{1}{|c|}{ Description } & Score \\
\hline A & Medium complexity of required maintenance & 2 \\
\hline B & Life support & 9 \\
\hline C & Medium risk & 2 \\
\hline D & High importance of the mission & 3 \\
\hline E & Old equipment & 2 \\
\hline G & Criticality= A + B + C + D + E & 18 \\
\hline
\end{tabular}




TABLE 6. Presence of Required Skills Through
Maintenance Level (1 if Yes, 0 if Not)
\begin{tabular}{|l|c|c|c|c|c|}
\hline StrategieslLevels & L1 & L2 & L3 & L4 & L5 \\
\hline Competence & 1 & 0 or 1 & 0 or 1 & 0 or 1 & 0 \\
\hline
\end{tabular}

checks that are carried out by internal technicians (included in the calculation of internal loads), and level L5 is the rare work requiring the intervention of the manufacturer (external). The decision to internalize concerns maintenance levels L2, L3, and L4 (Table 6).

\section{Estimated Annual Workload per Level per Maintenance Strategy per Device (I)}

For each level of maintenance, we define the annual workload in hours over the considered maintenance strategy. Note that CBM is present only for level L4 (Table 8), and the TBM is present for all levels. Table 9 contains the estimation of this criterion for the same equipment "anesthesia ventilator" given in Table 7 .

\section{Team per Level $(J)$}

For each maintenance level, we define the associated team composed of operators and engineers. Table 8 shows the team composition for the maintenance of the anesthesia ventilator.

\section{Cost per Hour (K)}

The cost per hour is determined based on the type of maintenance strategy, whether it is carried out by internal or external resources and the qualification of the personnel involved (operators and engineers).

We assume that for each device a potential subcontractor is already identified. Thus, for each device, we have the costs offered by the potential subcontractor. It is clear that a subcontractor may have several devices supported. The labor hour wage by subcontractors is the same regardless of the device. The in-house wage per hour is much cheaper than the outsourcing wages (Table 9).

\section{Labor Cost (L)}

The labor cost is determined by the multiplication of estimated maintenance workload (I) with the hourly wage of labors $(\mathrm{J})$ and the type and number of personnel $(\mathrm{K})$ involved

$$
L=I \times J \times K
$$

Internalization is done according to the feasibility in terms of competence and available tools. The in-house capacity is limited and considered as an input parameter. We propose a 3-phased simple heuristic to internalize levels that have high added value in terms of cost savings of insourcing compared with outsourcing. ${ }^{8}$ The proposed procedure works as follows:

Phase 1: Calculate the workload due to the internalization of L1 level for all devices (workload L1) and deduce the remaining capacity as Equation 5:

Remaining capacity $=$ internal capacity - workload L1

Phase 2: Classify the maintenance levels (L2, L3, and L4) of devices on which we have in-house expertise following descending order of (average) external cost / hour, then the (average) workload since the equipment maintenance. Strategy is not yet in this step, we take the average external cost per hour and the workload of the 3 possible strategies as estimation.

In Table 10, we describe how the equipment's levels are juxtaposed and how the 2 criteria are used to decide

TABLE 7. Estimated Annual Total Maintenance Hours per Level per Maintenance Strategy for Anesthesia
Ventilator Dräger Julian
\begin{tabular}{|l|c|c|c|c|}
\hline StrategieslLevels & L1 & L2 & L3 & L4 \\
\hline Time-based maintenance (G $=3)$ & 1 & 2 & 3 & 7 \\
\hline Condition-based maintenance (G = 2) & & & & 3 \\
\hline Corrective maintenance (G $=1)$ & 10 & 11 & 15 & 8 \\
\hline
\end{tabular}

TABLE 8. Team Composition per Level for the Maintenance if the Anesthesia Ventilator Dräger Julian (Operators, Engineers)

\begin{tabular}{|l|c|c|c|c|}
\hline StrategiesILevels & N1 & N2 & N3 & N4 \\
\cline { 1 - 3 } Time-based maintenance $(G=3)$ & $\{1,0\}$ & $\{2,0\}$ & $\{1,1\}$ & $\{1,1\}$ \\
\cline { 1 - 3 } Condition-based maintenance $(G=2)$ & & & & $\{1,1\}$ \\
\cline { 1 - 3 } Corrective maintenance $(G=1)$ & $\{1,0\}$ & $\{1,0\}$ & $\{2,1\}$ & $\{0,2\}$ \\
\hline
\end{tabular}


TABLE 9. Cost per Hour (in TND [Tunisian Dinar]) per Qualication per Subcontractor

\begin{tabular}{|l|c|c|c|c|c|}
\hline \multirow{2}{*}{} & \multirow{2}{*}{ In-house } & \multicolumn{2}{|c|}{ Subcontractor 1 } & $\cdots$ & \multicolumn{2}{c|}{ Subcontractor $p$} \\
\cline { 2 - 6 } & Equipment 1 & Equipment 14 & $\ldots$ & $\ldots$ \\
\hline Operator (TND) & 3.5 & 7 & $\ldots$ & 6 \\
\hline Engineer (TND) & 6 & 30 & $\ldots$ & 28 \\
\hline
\end{tabular}

whether to internalize or internalize the equipment's maintenance levels.

Phase 3: Decide to internalize the equipment's levels one by one following the order defined in phase 2, checking each time the feasibility workload versus remaining capacity of operator $(\mathrm{OP})$ (Equation 6) and engineer (ENG) (Equation 7):

$$
\begin{gathered}
\sum_{i=1}^{4=n} \mathrm{X}_{i}{ }^{*} \mathrm{wo}_{i} \leq \mathrm{OP} \text { remaining capacity } \\
\sum_{i=1}^{4^{*} n} \mathrm{X}_{i}{ }^{*} \mathrm{we}_{i} \leq \mathrm{ENG} \text { remaining capacity }
\end{gathered}
$$

Where $\mathrm{n}$ is the number of medical equipment, and 4 is the number of levels, $\mathrm{wo}_{j}\left(\mathrm{we}_{j}\right)$ is the workload of operators (engineers) per column $i$ (Table 12 ), and $X_{i} i$ s equal to 1 if equipment's level in column $i$ is internalized, 0 else.

For remaining equipment's levels (the ones for which we have in-house competence but decided not to carry out inside and the ones for which we do not have in-house competence), we apply a procedure for outsourcing and setting up contracts that we detail in the fourth step.

The real equipment maintenance cost will be defined exactly while contracts for externalized equipment will be defined in step 4; at this step and after the equipment maintenance levels are decided, we can have an estimation of the equipment maintenance cost (see Table 11 for the anesthesia ventilator) per maintenance strategy, which helps service maintenance to negotiate contract costs.

\section{Third Step: Dening the Equipment's Maintenance Strategy}

The objective of this section is to determine the maintenance strategy (TBM, CBM, or corrective maintenance) to be considered for each of the medical equipment based on the calculation of its criticality. We propose 3 classes (Table 12) with related strategies.

The thresholds T1 and T2 between criticality classes are defined based on the budget that is allocated to maintain

\section{TABLE 10. Procedure for Internalization/Externalization by Maintenance Level per Equipment per Year}

\begin{tabular}{|l|c|c|c|c|c|c|c|}
\hline Equipment, Level & $1 \mathrm{E}=1, \mathrm{~L} 4$ & $2 \mathrm{E}=4, \mathrm{~L} 3$ & $\ldots$ & $\mathrm{j} E=10, \mathrm{~L} 3$ & $\mathrm{j}+1 \mathrm{E}=20, \mathrm{~L} 2$ & $\cdots$ & 4 * $\mathrm{nE}=\mathrm{n}, \mathrm{L} 4$ \\
\hline Team composition & $\{0,2\}$ & $\{0,1\}$ & $\ldots$ & $\{0,2\}$ & $\{1,1\}$ & $\ldots$ & $\{2,1\}$ \\
\hline Operator workload $\left(w_{i}\right)$ & $0 \mathrm{~h}$ & $0 \mathrm{~h}$ & $\ldots$ & $0 \mathrm{~h}$ & $20 \mathrm{~h}$ & $\cdots$ & $20 \mathrm{~h}$ \\
\hline Engineer workload $\left(w e_{i}\right)$ & $40 \mathrm{~h}$ & $30 \mathrm{~h}$ & $\cdots$ & $50 \mathrm{~h}$ & $20 \mathrm{~h}$ & $\cdots$ & $10 \mathrm{~h}$ \\
\hline External cost/hour (TND) & 105 & 105 & $\ldots$ & 60 & 60 & $\cdots$ & 35 \\
\hline Internalized $\left(X_{i}=\{0,1\}\right)$ & 1 & 1 & $\cdots$ & 0 & 1 & $\cdots$ & 0 \\
\hline
\end{tabular}

TABLE 11. Cost per year (in TND) of the Anestesia Ventilator Maintenance per Strategy

Anesthesia Ventilator Dräger Julian

\begin{tabular}{|l|c|c|c|c|}
\hline \multirow{2}{*}{} & \multicolumn{4}{|c|}{ Anesthesia Ventilator Dräger Julian } \\
\cline { 2 - 3 } & Externalized Levels Workload Costs (TND) & \multirow{2}{*}{$\begin{array}{c}\text { Estimation of Spare } \\
\text { Parts Costs (TND) }\end{array}$} & $\begin{array}{l}\text { Total Cost of the } \\
\text { Maintenance Strategy }\end{array}$ \\
\cline { 2 - 5 } & L3 & L4 & $10,190.56$ & $10,672.06$ TND \\
\hline Time-based maintenance & 206 & 275.5 & $7,674.63$ & $8,091.38$ TND \\
\hline Condition-based maintenance & 162.25 & 254.5 & 4,838 & 5,281 \\
\hline Corrective maintenance & 230 & 213 & & \\
\hline
\end{tabular}


TABLE 12. Different Levels of Criticality and Strategies Relevant Maintenance

\begin{tabular}{|l|c|c|}
\hline Strategies & Criticality & Score \\
\hline Time-based maintenance $(\mathrm{G}=3)$ & Criticality $\geq \mathrm{T} 2$ & High \\
\hline Condition-based maintenance $(\mathrm{G}=2)$ & $\mathrm{T} 1 \leq$ criticality $<\mathrm{T} 2$ & Low \\
\hline Corrective maintenance $(\mathrm{G}=1)$ & Criticality $<\mathrm{T} 1$ & Low \\
\hline
\end{tabular}

these devices and the available resources (Table 13 ). In fact, corrective maintenance strategy does not require any preplanned resources (because it is applied for noncritical devices). However, TBM requires resources and a reserved budget. Finally, the CBM strategy is in between and requires special tools.

We have seen in step 1 that the total score of criticality is between 5 and 20. The thresholds T1 and T2 are between 5 and 20 too, whereas T2 is greater than T1. In addition, the total maintenance cost should be less than or equal to the budget. The total cost varies according to the maintenance strategies that we will define for the equipment. Thus, it depends on the values of the threshold T1 and T2 $\left(\operatorname{cost}_{\mathrm{T} 1 \mathrm{~T} 2}=f(\mathrm{~T} 1, \mathrm{~T} 2)\right)$. Matrix in Table 14 contains an estimation of the total maintenance costs according to the values of T1 and T2; for example, $\mathrm{C}_{5,6}$ is the cost, whereas time-based maintenance is applied for all equipment.

The objective is to apply preventive maintenance to the maximum number of equipment without exceeding the budget. In case of Habib Bourguiba Hospital, we proposed values of T1 and T2 equal to 10 and 15, respectively, which corresponds to the nearest total cost value to the budget with the lower value of T2. ${ }^{22}$ The total cost is $C_{10,15}=$ 14,850,000 TND (Tunisian dinar), where the budget is equal to 1,500,000 TND. The maintenance strategy for anesthesia ventilator, in particular, which criticality is equal to 18 (Table 11), is the TBM strategy, and the corresponding maintenance cost is equal to 95,000 TND. For confidentiality, values shared here are not the real ones.

\section{Fourth Step: Outsourcing and Setting Up the Contract}

Four types of contracts are used in Tunisia and particularly in Habib Bourguiba Hospital: A*, A, B, and C. For all these contracts, clauses on equipment availability (at least $95 \%$ ) and response time (at most 72 hours) are the same. The difference lies in the selected maintenance strategy and the package constitution in terms of spare parts and labor (see first part of the article). According to de Melo Brito et $\mathrm{al},{ }^{23}$ the selection of contracts is a very important step for the outsourcing process in the current trend toward reducing costs.

A procedure for choosing the type of contract based on the previous results is provided in the following. This procedure is based on 4 criteria: The criticality $(G)$, the cost of labor $(\mathrm{L})$, the monetary value of spare parts $(\mathrm{F})$, and the frequency of complex failures $(\mathrm{O})$.

The first 2 criteria $G$ and $L$ are already explained in the previous steps. We just classify the labor cost (L) into 2 possible levels (high with score $=2$ and low with score $=1$ ). Similarly, we consider 2 levels for the 2 other criteria. The labor cost (L) criterion allows us to choose between 2 types of contract looking at whether they include labor in the package. For example, if we limit the choice between contracts A and B and the medical equipment requires a high labor cost (skilled labor), we choose contract $\mathrm{A}$; otherwise, we choose contract $\mathrm{B}$.

\section{Monetary Value of Spare Parts (F):}

This criterion allows us to choose between 2 types of contracts looking at whether they include spare parts in the package. For example, if we limit the choice between contracts $\mathrm{B}$ and $\mathrm{C}$, and the medical device has a high spare parts cost, we choose contract B; otherwise, we choose contract C.

\section{Frequency of Complex Failures $(\mathrm{O})$ :}

This criterion indicates the occurrence of unexpected complex failures. It allows us to choose between 2 types of contracts looking at whether they include corrective maintenance in the package. For example, if we limit the choice between contracts $\mathrm{A}$ * and $\mathrm{A}$, and the medical device exhibits a high frequency of complex failures, we choose contract $\mathrm{A}^{*}$; otherwise, we choose contract $\mathrm{A}$.

The preferred type of contract is thus defined for maintenance levels L2, L3, and L4 for all equipment based on criteria G, F, L, and O (Table 15). This allows us to choose the type of contract for the equipment according to a

\begin{tabular}{|l|l|l|}
\hline TABLE 13. & $\begin{array}{l}\text { Denition of Thresholds per Required } \\
\text { Resources, Cost, Labor, and Tools for } \\
\text { Maintenance Strategies (High } \uparrow \text {, Low } \downarrow \text { ) }\end{array}$ \\
\hline \multirow{3}{*}{ Corrective Maintenance } & \multicolumn{2}{|l|}{ Preventive Maintenance } \\
\cline { 2 - 3 } & CBM & TBM \\
\hline Resources $\downarrow$ & Resources $\uparrow$ & \\
\hline & Budget $\downarrow$, tools $\uparrow$ & Budget $\uparrow$, tools $\downarrow$ \\
\hline
\end{tabular}


TABLE 14. Upper Triangular Matrix of Total Maintenance Cost Depending on Thresholds (T1, T2)

\begin{tabular}{|c|c|c|c|c|c|c|c|c|c|}
\hline T2 T1 & 5 & 6 & 7 & 8 & $\ldots$ & 17 & 18 & 19 & 20 \\
\hline 5 & $C_{5,5}=f(5,5)$ & $C_{5,6}=f(5,6)$ & . & . & $\ldots$ & . & . & . & $C_{5,20}=f(5,20)$ \\
\hline 6 & 0 & $C_{6,6}=f(6,7)$ & & . & $\ldots$ & & . & . & \\
\hline 7 & 0 & 0 & . & . & $\ldots$ & . & . & . & . \\
\hline 8 & . & & . & . & $\ldots$ & & . & . & \\
\hline 9 & v & . & . & . & $\ldots$ & & . & . & \\
\hline$\cdots$ & $\cdots$ & $\cdots$ & $\cdots$ & $\cdots$ & $\ldots$ & $\cdots$ & $\cdots$ & $\cdots$ & $\cdots$ \\
\hline 18 & . & . & . & . & $\cdots$ & & . & . & \\
\hline 19 & . & . & . & . & $\ldots$ & . & . & . & . \\
\hline 20 & 0 & 0 & . & . & $\ldots$ & & 0 & 0 & $C_{20,20}=f(20,20)$ \\
\hline
\end{tabular}

certain degree of risk and to define the content of contract in relation to the different levels of maintenance in terms of labor and spare parts. For example, we take the same equipment "anesthesia ventilator" given before, and we apply our proposed procedure to select the appropriate contract for this equipment. For level L1, the activities are internalized. For levels L2 and L3, the activities are externalized with contract type A, and for level L4, the activities are externalized using contract type A*. Finally, there are 2 possible choices:

Contract type A* in which the package constituted of preventive maintenance and associated spare parts for maintenance levels L2, L3, and L4 and also corrective maintenance and associated spare parts only for L4 level

Contract type A in which there will only be preventive maintenance and associated spare parts for maintenance levels L2, L3, and L4, without corrective maintenance

A certain degree of risk is to consider in case where subcontractors offer contracts with rigid formulas. Our procedure allows us to take the best decisions with the minimum of risk.

For hospitals in developed countries, biomedical engineering maintenance services are contracted out to private companies. This decision to contract out is almost due to the high-end technology used in the medical equipment and also the unavailability of internal expertise. However, in developing countries, the contract is covering labor and spare parts. Therefore, clauses and types of contracts for developed and developing countries are different.

The provided procedure is quite generic. It can be applied for hospitals of developed countries with some differences in definition of thresholds and final contracts types; for example, the mean age of the equipment can be more or less than 10 years. Partenarial contract can be added. ${ }^{10}$

\section{Conclusions and Future Research}

In this article, we provide a procedure to define maintenance strategy per medical device, to choose outsourcing or insourcing maintenance levels for all devices and to choose the appropriate contract for each device based on multicriteria with levels that we validated with experts. We explored how the budget should be managed, and the contract type could be negotiated with the subcontractor. We plan to implement this decision support procedure in the software for biomedical maintenance management called Biomed that is used in all hospitals in Tunisia. Making a proposal to improve the module "Contract

\begin{tabular}{|l|c|}
\hline \multicolumn{1}{|c|}{ TABLE 15. $\begin{array}{c}\text { Selecting Contract Based on Criteria G, } \\
\text { Multicriteria }\end{array}$} \\
\hline$G=3, L=2, O=2$ & Contract Type \\
\hline$G=3, L=2, O=1$ & A \\
\hline$G=3, L=1, F=2$ & $B$ \\
\hline$G=3, L=1, F=1$ & $C$ \\
\hline$G=2, L=1, F=2$ & $B$ \\
\hline$G=2, L=1, F=1$ & $C$ \\
\hline$G=1, L=1, F=2$ & $C$ \\
\hline$G=1, L=1, F=1$ & No contract \\
\hline
\end{tabular}


Management: Selection and Monitoring" of this software with the involvement of health managers and software developers is in our perspective. In view of the need for more structured and robust contract selection procedure, we will focus on developing methodologies for contract choice based on quantitative multicriteria approaches. Then, we will focus on the development of mathematical models for minimizing future costs to build it into the contract price and to increase the availability of medical equipment. This will be done by integrating the cost of tools, the cost associated with maintenance strategies, and failure modes of equipment.

\section{Acknowledgment}

The authors thank the staff at Biomedical Maintenance Service of Habib Bourguiba Hospital for their cooperation in the development of this research.

\section{References}

1. Jamshidi A, Abbasgholizadeh RS, Ait-kadi D, Ruiz A. Medical devices inspection and maintenance; a literature review. Proceeding of the 2014 Industrial and Systems Engineering Research Conference.

2. Khalaf AB. Maintenance model for minimizing risk and optimizing costeffectiveness of medical equipment in Palestine. J Clin Eng. 2004;14: 3649-3653.

3. Letaief F, Kammoun A, Khayat S, et al. Manuel des Procédures de Gestion de la Maintenance Biomédicale et Hospitalière, Ministre de la santè publique de Tunisie, Vol. III. 2007.

4. Mkalaf K, Gibson P, Flanagan J. A study of current maintenance strategies and the reliability of critical medical equipment in hospitals in relation to patient outcomes, World Academy of Science, Engineering and Technology, International Science Index 82. Int J Soc Hum Sci Eng. 2013;7(10):15-22.

5. Rahman A, Chattopadhyay G. Optimal service contract policies for outsourcing maintenance service of assets to the service providers. Int J Reliability Appl. 2007;8(2):183-197.

6. Taghipour S, Banjevic D, Jardine AKS. Prioritization of medical equipmentment for maintenance decisions. J Oper Res Soc. 2011;62(9):1666-1687.

7. Gits CW. Design of maintenance concepts. Int J Prod Econ. 1992;24:217-226.

8. Walraeve B, Vigneau P. Guide pratique, Maintenance des dispositifs médicaux, obligations et recommandations. Direction régionale des affaires sanitaires et sociales de Midi-Pyrénées, Conception-réalisation: Drass midi-Pyrénéesmars. Toulouse, France; 2005.

9. Cruz AM, Rincon AMR. Medical device maintenance outsourcing: have operation management research and management theories forgotten the medical engineering community? A mapping review. Eur J Oper Res. 2012; 221(1):186-197.

10. De Vivo L, Derrico P, Tomaiuolo D, Capussotto C, Reali A. Evaluating alternative service contracts for medical equipment. Presented at the 26th Annual International Conference of the IEEE EMBS; San Francisco, CA; September 1-5, 2004.

11. Cruz AM, Perilla SP, Pabón NN. Clustering techniques: measuring the performance of contract service providers. IEEE Eng Med Biol Mag. 2010;29(2):119-126.

12. Association for the Advancement of Medical Instrumentation (AAMI, 1985). Guidelines for the Establishing and Administering Medical Instrumentation Maintenance Programs. USA. http://www.aami.org/. Accessed November 27, 2015.

13. Khalaf AB, Djouani K, Hamam Y, Alayli Y. Evidence-based mathematical maintenance model for medical equipment. In: Electronic Devices, Systems and Applications (ICEDSA). 2010.

14. Fennigkoh L, Smith B. Clinical equipment management. JCAHO Ptsm Ser. 1989;2:5-14.

15. Wang B, Levenson A. Equipment inclusion criteria-a new interpritation of JCAHO's medical equipment management standard. J Clin Eng. 2000; 25(1):26-35.

16. Jamshidi A, Abbasgholizadeh RS, Ait-kadi D, Ruiz A. A risk-based maintenance strategy using Fuzzy HFMEA for prioritization of critical medical equipment. Presented at the 1st Annual World Conference of the Society for Industrial and Systems Engineering; Washington, DC; September 16-18, 2012.

17. Khalaf AB, Hamam Y, Djouani K. Mathematical Maintenance Model for Medical Equipment, University of Versailles Saint-Quentin-en-Yvelines. Versailles, France; Scholar's Press; 2012.

18. Georgin D, Natan J, Szymczak H, Farges G. Management de l'externalisation de la maintenance biomédicale. ITBM RBM News. 2005;26(6):7-11.

19. Panayiotou NA, Ponis ST, Gayialis SP. Designing an industrial maintenance system: a proposed methodological framework. Int J Inform Technol Manage. 2009;8(4):361-381.

20. Rzevsky G. Mechatronics: Designing Intelligent Machines. ButterworthHeinemann: England; 1995.

21. Norme NF-X60-010. Maitenance. Concepts et définitions des activités de maintenance. 1994.

22. Masmoudi M, Ben Houria Z, Masmoudi F. Multi-criteria decision making for Medical equipment maintenance: insourcing, outsourcing and service contract. Presented at the IEEE, 2nd International Conference on Control, Decision and Information Technologies, CODIT'2014; Metz, France; November 3-5, 2014.

23. de Melo Brito AJ, Almeida A. Multi-criteria decision model for selecting repair contracts by applying utility theory and variable interdependent parameters. J Manage Math. 2010;21:349-361. 